\title{
Complete response of a hepatocellular carcinoma with complex macrovascular invasion after combined treatment with chemoembolization and immunotherapy: a case report
}

\author{
A.-M. Bucalau', I. Tancredi' ${ }^{2}$ M. Pezzullo ${ }^{3}$, A. Covas ${ }^{4}$, G. Verset ${ }^{1}$ \\ (1) Department of Gastroenterology, Hepatopancreatology and Digestive Oncology, Hôpital Erasme, Université libre de Bruxelles (ULB), Brussels ; (2) Department of \\ Interventional Radiology, Hôpital Erasme, Brussels ; (3) Department of Radiology, Hôpital Erasme, Brussels ; (4) Department of Gastroenterology, Marie Curie Hospital, \\ Charleroi.
}

\begin{abstract}
Hepatocellular carcinoma accounts for $90 \%$ of primary liver cancers and represents a growing health problem worldwide. We report the complex case of a 71 year-old patient diagnosed with a large hepatocellular carcinoma and presenting an extensive vascular invasion of the middle hepatic vein and the inferior caval vein ascending to the right atrium with no extrahepatic spread. Due to several comorbidities, a systemic treatment by tyrosine kinase inhibitors was contraindicated. After discussion at the multidisciplinary hepatology tumor board, he was referred for selective internal radiation therapy. Unfortunately, the work-up showed an important lung shunt not allowing radioembolization. No clear recommendations are available in this situation. The decision was made to propose a combination treatment by transarterial chemoembolization, that was performed using a new generation of radio-opaque microspheres loaded with doxorubicin, followed by immunotherapy. This allowed a complete response with a very good quality of life. (Acta gastroenterol. belg., 2021, 84, 371-374).
\end{abstract}

Keywords : Transcatheter arterial chemoembolization (TACE), selective internal radiation therapy (SIRT), hepatocellular carcinoma (HCC), immunotherapy.

\section{Introduction}

Hepatocellular carcinoma (HCC) accounts for $90 \%$ of primary liver cancers with the majority of cases being associated to cirrhosis (1). The last few decades have seen the emergence of non-viral causes of HCC, the most important being non-alcoholic fatty liver disease (NALFD) $(2,3)$. Current guidelines $(1,4)$ consider the Barcelona Clinic Liver Cancer (BCLC) staging system (5) as the algorithm of choice for tumor staging and therapeutic options.

Locally advanced HCC (BCLC B) is suitable for transarterial locoregional therapies that take advantage of the double vascularization of the liver, with $75 \%$ of the parenchyma's blood supply coming from the portal vein, while tumor nodules' blood supply being almost exclusively provided by the hepatic artery (6).

Transarterial chemoembolization (TACE) is recommenced as first-line treatment for these patients $(1,4)$. Nevertheless, during the last decade, selective internal radiation therapy (SIRT) with yttrium-90 microspheres (Y90) was introduced in case of TACE failure or for patients who were not suitable for TACE (i.e.: large tumors). Moreover, advanced HCC (BCLC C) presenting a macrovascular invasion and no extrahepatic spread may also constitute an indication for radioembolization when systemic treatment (e.g. tyrosine kinase inhibitors, TKI) is contraindicated $(7,8)$. Its efficacy was reported in several large patient series (9-12).

We report here the case of a 71 year-old patient presenting a large hepatocellular carcinoma with an extensive vascular invasion of the middle hepatic vein and the inferior caval vein. A treatment by SIRT was initially proposed, but not feasible due to an important lung shunt. No clear recommendations are available in this situation. Combined treatment with TACE followed by immunotherapy with Nivolumab achieved complete response.

\section{Case report}

This is the case of a 71 year-old male with a personal history of ischemic cardiomyopathy already treated by coronary angioplasty and stenting, aortic valve stenosis, anticoagulated atrial fibrillation and chronic obstructive pulmonary disease (COPD). He also suffered from a chronic lower limb ischemia for which he underwent multivessel angioplasty. He presented multiple cardiovascular risk factors such as diabetes, arterial hypertension, dyslipidemia and obesity. He was a former smoker and presented an alcohol consumption of 2 units per week.

In December of 2018 he presented right upper abdominal pain and the abdominal ultrasound showed a large hypoechoic lesion in the segment 4 , hepatic steatosis and multiples gallbladder stones. In order to better characterize this lesion, an abdominal magnetic resonance imaging (MRI) was performed. It showed a neoplastic tissue within the lumen of the middle hepatic artery going into the inferior caval vein and ascending to the right atrium, presenting the typical imaging characteristics of HCC, more precisely arterial hyperenhancement and portal vein/delayed phase wash-

Correspondence to : Ana-Maria Bucalau, Hôpital Erasme, Route de Lennik 808, 1070 Brussels, Belgium

Email : Ana-Maria.Bucalau@erasme.ulb.ac.be

Submission date : 06/05/2020

Acceptance date : 16/08/2020 

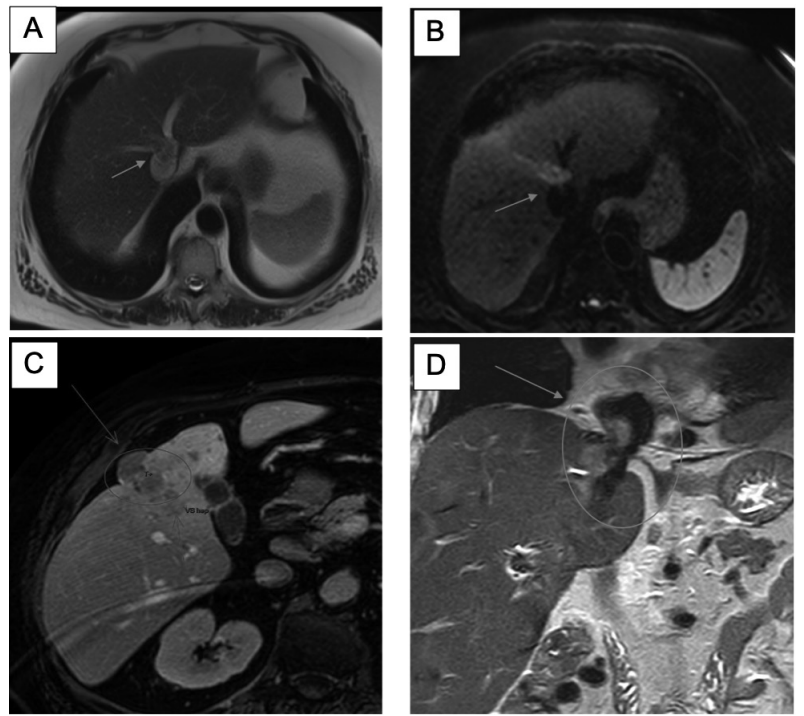

Figure 1. - Baseline imaging by magnetic resonance (MRI).

out (Fig. 1 A-D). Furthermore, the MRI disclosed signs of cirrhosis, probably due to a non-alcoholic fatty liver disease (NAFLD) (i.e. diabetes, arterial hypertension and obesity). The chest computed tomography (CT) and the bone scintigraphy disclosed no extrahepatic spread. Serum alpha protein (AFP) was elevated at 20,000 micrograms/liter and the rest of the blood tests showed a preserved liver function. After discussion in the multidisciplinary hepatology tumor board, he was referred for SIRT due to the contraindications for TKI treatment. During the work-up, a hepatic angiogram was performed. Selective opacification of the right hepatic artery showed a good vascular blush in segment 4, corresponding to the tumor previously describes, with a complex arterial tumoral neovascularizationmiddle hepatic vein fistula and early opacification of the middle hepatic vein (Fig. 2 A). Furthermore, in the portal venous phase, an opacification of the right and middle hepatic veins was noted suggesting multiples small collateral vessels, as well as an enhancement of the tumor ascending to the hepatic vein confluence (Fig. 2 B). This finding suggested an important lung shunting, motivating a prophylactic embolization of the middle hepatic vein. This was performed percutaneously, under local anesthesia and ultrasound guidance (Fig. 2 C). The mid-hepatic vein was punctured directly and occluded by coils. Opacification through the microcatheter placed proxi-mally in segment 4 showed an increased uptake in the tumor and a disappearance of the hepatic vein opacification (Fig. 2 D). Technetium 99m-labeled macroaggregated albumin (Tc-99m-MAA) was subsequently administered and the patient underwent single photon emission computed tomography (SPECT/CT) imaging of the liver within the hour. Unfortunately, important lung shunting was visualized contraindicating SIRT. Nevertheless, the tumor uptake was favorable and a second work-up was attempted, in order to improve the occlusion of the middle hepatic vein and diminish the
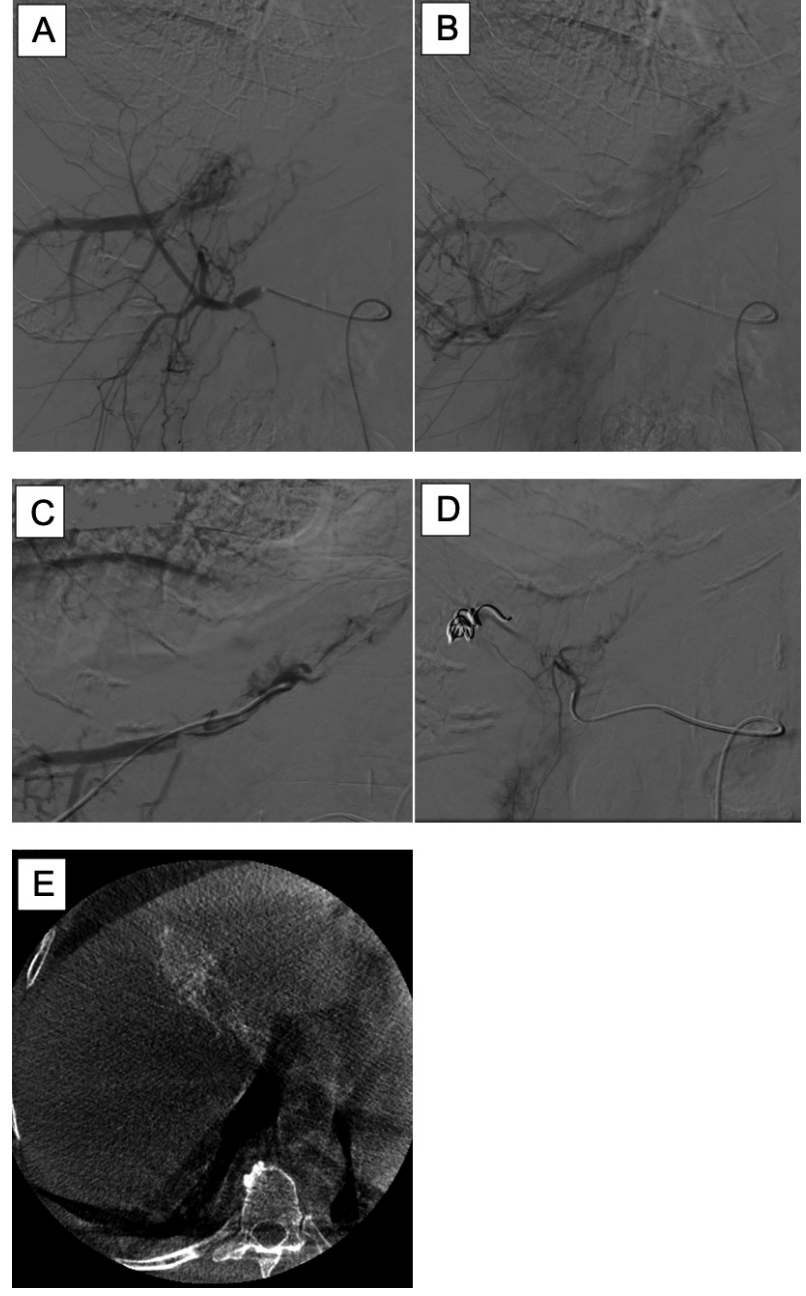

Figure 2. - Locoregional transarterial therapies.

lung shunting. A vascular plug was placed downstream of the coils and the microcatheter was place in the same position as for the first work-up considering the good tumor uptake. No shunting was angiographically highlighted, nevertheless the SPECT/CT imaging still showed an important lung uptake. Considering the well vascularized nature of the tumor, chemoembolization was proposed with the new radio-opaque microspheres loaded with doxorubicin $75 \mathrm{mg}$ (Boston Scientific, Los Angeles, USA). TACE was performed through right femoral access under local anesthesia by the same interventional radiologist that performed the two SIRT work-ups. The three-dimensional computed tomography (cone-beam CT) performed after TACE displayed a very good tumor targeting (Fig. 2 E). An abdominal MRI was performed at one-month post treatment and showed a partial response of the tumor according to the modified response evaluation criteria in solid tumors (mRECIST) (13), although persistent intraluminal vascularized tissue was still present. Serum AFP had decreased to 14,000 micrograms/liter.

Due to this incomplete response a systemic treatment was proposed and the patient was started on immunotherapy with Nivolumab 3 milligrams/kilogram every 
two weeks. Tolerance was excellent, no adverse events were reported.

The MRI performed 6 months after the start of the treatment showed size decrease of the tumor and no evidence of vascular residual tissue was seen within the targeted lesion. Serum AFP was also normalized $(<3$ micrograms/liter).

\section{Discussion}

Hepatocellular carcinoma with vascular invasion of the hepatic veins and inferior naval vein is rare. According to current recommendations $(1,4)$, TACE can be proposed in several situations: for intermediate stages (BCLC B), for early stages (BCLC A) which are contraindicated to curative treatments, for downstaging or as bridge to liver transplantation. Unlike TACE, the place of SIRT has yet to be defined. In fact, two recent phase 3 clinical studies have evaluated the impact on the overall survival of SIRT versus sorafenib in an advanced stage population, the latter being one of the first line of systemic treatment in HCC. These studies have shown that there is no difference in terms of overall survival between these two treatments $(14,15)$. However, these studies have a significant technical limit linked to an imprecise dosimetry leading to an under assay of the radioactive component within the tumor.

Regarding our patient, SIRT was considered the most suitable treatment due to its comorbidities. Unfortunately, the important lung shunting did not allow treatment with radioembolization. The use of TACE in patients with $\mathrm{HCC}$ and vascular invasion (BCLC C) is still considered a contraindication due the risk of complications after the procedure, including acute liver failure or intrahepatic tumor progression. Nevertheless, a systematic review showed that chemoembolization could be considered as a treatment for selected advanced HCC patients with vascular invasion and adequate liver function with a gain in overall survival, that ranges from 4 to 14 months, and good tolerance (16). In our case there is no portal vein invasion, which limits the risk of liver decompensation.

Due to the volume of the tumor and the vascular invasion it was decided to combine two treatment modalities, thus TACE was followed by immunotherapy with Nivolumab. This concept derived from a recent study that combined immune checkpoint inhibition treatment (anti CTLA-4 1antibody tremelimumab) with subtotal TACE or ablation in patients with advanced HCC and showed a duration of response between 7 and 19 months, with a good tolerance (17). This association is based on the assumption that ablation therapy induces an immune response that could be potentially amplified by immune modulating agents. In this setting, a Phase 2 trial is ongoing, associating CTLA-4 /PD-L1 (tremelimumab and durvalumab) blockade following TACE in patients with intermediate stage HCC (BCLC B) (NCT03638141). The last decade has led to a better understanding of molecular abnormalities in HCC, that together with specific target treatments will lead to a personalized treatment for HCC (18).

\section{Conclusion}

Our case presents a complex and rare hepatocellular carcinoma with an extensive vascular invasion of the middle hepatic vein and inferior caval vein and thus considered as a palliative situation. Nevertheless, he was successfully treated with TACE followed by immunotherapy and achieved complete radiological response and normalization of serum AFP levels.

\section{Conflict of interest}

Dr. AM. Bucalau has nothing to disclose ; Dr. I. Tancredi reports grants from Terumo, other from Terumo, other from BTG, outside the submitted work ; Dr. M. Pezzullo has nothing to disclose ; Dr. A. Covas has nothing to disclose; Dr. G. Verset reports grants from Terumo, other from Terumo, other from BTG, other from Bayer, other from Ipsen, other from BMS, other from Lilly, other from Eisai, other from Gilead, other from Merck serono, outside the submitted work.

\section{References}

1. GALLE P. R., FORNERA., LLOVET J. M., MAZZAFERRO V., PISCAGLIA F., RAOUL J. L. et al. EASL Clinical Practice Guidelines: Management of hepatocellular carcinoma. J. Hepatol. 2018, 69(1) : 182-236.

2. LEFERE S., DEVISSCHER L., GEERTS A. Angiogenesis in the progression of non-alcoholic fatty liver disease. Acta Gastroenterol. Belg. 2020, 83(2) : 301-307.

3. FRANCQUE S., LANTHIER N., VERBEKE L., REYNAERT H., VAN STEENKISTE C., VONGHIA L. et al. The Belgian Association for Study of the Liver Guidance Document on the Management of Adult and Paediatric Non-Alcoholic Fatty Liver Disease. Acta Gastroenterol. Belg. 2018, 81(1) : $55-81$

4. HEIMBACH J. K., KULIK L. M., FINN R. S., SIRLIN C. B., ABECASSIS M., ROBERTS L. R. et al. AASLD guidelines for the treatment of hepatocellular carcinoma. Hepatology. 2018, 67(1) : 358-380.

5. FORNER A., REIG M., BRUIX J. Hepatocellular carcinoma. Lancet. 2018 , 391(10127) : 1301-1314

6. BREEDIS C., YOUNG G. The blood supply of neoplasms in the liver. Am. $J$ Pathol. 1954, 30(5) : 969-977.

7. SANGRO B., CARPANESE L., CIANNI R., GOLFIERI R., GASPARINI D., EZZIDDIN S. et al. Survival after yttrium-90 resin microsphere radioembolization of hepatocellular carcinoma across Barcelona clinic liver cancer stages : a European evaluation. Hepatology. 2011, 54(3) : 868-878.

8. SALEM R., LEWANDOWSKI R. J., KULIK L., WAND E., RIAZ A., RYU R. K. et al. Radioembolization results in longer time-to-progression and reduced toxicity compared with chemoembolization in patients with hepatocellular carcinoma. Gastroenterology. 2011, 140(2) :497-507.e2.

9. SALEM R., MAZZAFERRO V., SANGRO B. Yttrium 90 radioembolization for the treatment of hepatocellular carcinoma : biological lessons, current challenges, and clinical perspectives. Hepatology. 2013, 58(6) : 2188-2197.

10. SALEM R., LEWANDOWSKI R. J., MULCAHY M. F., RIAZ A., RYU R. K., IBRAHIM S. et al. Radioembolization for hepatocellular carcinoma using Yttrium-90 microspheres: a comprehensive report of long-term outcomes. Gastroenterology. 2010, 138(1) : 52-64.

11. HILGARD P., HAMAMI M., FOULY A. E., SCHERAG A., MÜLLER S., ERTLE J.et al. Radioembolization with yttrium-90 glass microspheres in hepatocellular carcinoma: European experience on safety and long-term survival. Hepatology. 2010, 52(5) : 1741-1749.

12. MAZZAFERRO V., SPOSITO C., BHOORI S., ROMITO R., CHIESA C., MOROSI C. et al. Yttrium-90 radioembolization for intermediate-advanced hepatocellular carcinoma: a phase 2 study. Hepatology. 2013, 57(5) : 18261837. 
13. LENCIONI R., LLOVET J. M. Modified RECIST (mRECIST) assessment for hepatocellular carcinoma. Semin Liver Dis. 2010, 30(1) : 52-60.

14. VILGRAIN V., PEREIRA H., ASSENAT E., GUIU B., ILONCA A. D., PAGEAUX G. P. et al. Efficacy and safety of selective internal adiotherapy with yttrium-90 resin microspheres compared with sorafenib in locally advanced and inoperable hepatocellular carcinoma (SARAH): an open-label randomised controlled phase 3 trial. Lancet Oncol. 2017, 18(12) : 1624-1636. 15. CHOW P. K. H., GANDHI M., TAN S. B., WIN KHIN M., KHASBAZAR A., ONG J. et al. SIRveNIB: Selective Internal Radiation Therapy Versus Sorafenib in Asia-Pacific Patients With Hepatocellular Carcinoma. J. Clin. Oncol. 2018, 36(19) : 1913-1921.
16. ZHAO Y., CAI G., ZHOU L., LIU L., QI X., BAI M. et al. Transarterial chemoembolization in hepatocellular carcinoma with vascular invasion or extrahepatic metastasis: A systematic review. Asia Pac. J. Clin. Oncol. 2013, 9(4) : 357-364.

17. DUFFY A. G., ULAHANNAN S. V., MAKOROVA-RUSHER O., RAHMA O., WEDEMEYER H., PRATT D. et al. Tremelimumab in combination with ablation in patients with advanced hepatocellular carcinoma. J. Hepatol. 2017 66(3) : 545-551.

18. DEMORY A., NAULT J. C. Molecular perspectives for the treatment of hepatocellular carcinoma. Acta Gastroenterol. Belg. 2020, 83(2) : 309-312. 